Short Communication

\title{
Electrospinning of nanocomposite nanofibers from cyclodextrin and laponite
}

\author{
Fuat Topuz*, Tamer Uyar* \\ Institute of Materials Science \& Nanotechnology, UNAM-National Nanotechnology Research Center, Bilkent University, 06800 Ankara, Turkey
}

\section{A R T I C L E I N F O}

\section{Keywords:}

Cyclodextrin

Laponite

Clay

Electrospinning

Nanofibers

Nanocomposite

\begin{abstract}
A B S T R A C T
Herein, the electrospinning of nanocomposite nanofibers from a non-polymeric system using cyclodextrin (CD) and Laponite was reported. Laponite, a hectorite-type synthetic clay, was used as an additive in the aqueous solutions of CD molecules, and its influence on the polymer-free electrospinning of CD was investigated. Flow tests showed that the viscosity of CD solutions increased with a Laponite content rise at low shear rates and an unequalled degree of shear thinning at high shear rates. The morphology of the CD/Laponite nanofibers was explored by SEM, which revealed the formation of smooth nanofibers at low Laponite content $(0.62 \mathrm{wt} \%)$ and non-smooth nanofibers at Laponite content over $1.56 \mathrm{wt} \%$ due to enhanced electrostatic interactions between the charged surface of Laponite and hydroxyl groups of CD. Further increasing Laponite content to $12.5 \mathrm{wt} \%$ led to only micro-sized beads instead of fibers due to electrospraying, suggesting that the embedded Laponite significantly disturbed hydrogen bonds among CD molecules. The presence of Laponite in the nanofibers was confirmed over chemical analysis by EDX and XPS. TEM analysis displayed homogeneous distribution of the exfoliated Laponite in the CD nanofibers, as supported by the disappearance of the diffraction patterns of Laponite by WAXS. The nanocomposite CD/Laponite mats with Laponite content of $\leq 1.56 \mathrm{wt} \%$ maintained their self-standing and flexible structure to some extent, as revealed by tensile tests. In brief, this study reports the polymer-free electrospinning of nanocomposite nanofibers based on CD and Laponite and investigates the impact of the incorporated clay on the polymer-free electrospinning of CD molecules.
\end{abstract}

\section{Introduction}

Electrospun nanofibers have sparked considerable interest due to their high aspect ratio and tunable fiber properties for a wide spectrum of applications, including drug delivery [1], sensing [2], catalysis [3], sorption [4], tissue engineering [5-7] and fiber reinforced materials [8]. Generally, electrospinning process requires a polymeric carrier matrix to form continuous fibers, in which the presence of chain entanglements for high molecular weight molecules facilitate the formation of a stable jet during electrospinning. Yet, the last decade has also witnessed the polymer-free electrospinning of functional molecules, such as phospholipid [9], gemini ammonium surfactant [10], cyclodextrin (CD) derivatives [11-14] and tannic acid [15]. Likewise, native CDs ( $\alpha$-CD [16], $\beta$-CD [16], and $\gamma$-CD [17]) and CD-inclusion complexes $[18,19]$ were electrospun to obtain nanofibrous materials. The formation of continuous electrospun nanofibers from such small molecules (e.g., CD molecules) can be attributed to the existence of intermolecular interactions, such as hydrogen bonds as in the case of the electrospinning of CD molecules, which holds the molecules together under high shear force caused by electrospinning. Thus, the polymer-free electrospinning of CD molecules is highly sensitive to the presence of nanoparticles that can disturb intermolecular interactions (i.e., hydrogen bonds) between $\mathrm{CD}$ molecules, and hence, affects the fiber formation during the electrospinning process. In this regard, even though the presence of numerous studies on polymeric nanocomposite electrospun nanofibers, to the best of our knowledge, to date there has been no report on nanocomposite nanofibers produced from a non-polymeric system, such as cyclodextrin (CD) and nanoparticle (e.g., Laponite clay).

Because of their high aspect ratio, well-defined crystal structure and dissimilar charge distribution, Laponite particles have been widely exploited in bio-related and environmental applications [20-23]. Laponite (i.e., synthetic hectorite-type clay) has a chemical formula of $\mathrm{Na}_{0.7} \mathrm{Si}_{8} \mathrm{Mg}_{5.5} \mathrm{Li}_{0.3} \mathrm{O}_{20}(\mathrm{OH})_{4}$ and decorated with external pyrophosphate groups for Laponite ${ }^{\circ}$ RDS type so that both surfaces and edges are negatively charged. This allows the preparation of Laponite solutions at higher concentrations than other Laponite derivatives, which mostly have opposite charges on both faces and form highly viscous solutions even at very low concentrations. It has a disk-like shape with a diameter

\footnotetext{
* Corresponding authors.

E-mail addresses: fuat.topuz@rwth-aachen.de (F. Topuz), uyar@unam.bilkent.edu.tr (T. Uyar).
} 
of $25 \mathrm{~nm}$ and thickness of $1 \mathrm{~nm}$. Laponite has been used as an additive in numerous electrospun fiber systems to be benefited from their intrinsic bioactivity and cell adhesion features. In this regard, Laponiteembedded poly(lactic-co-glycolic acid) nanofibers have shown bioactivity by the osteogenic differentiation of human mesenchymal stem cells (hMSC) [24]. Due to its charged surface and dissolution products, i.e., $\mathrm{Mg}^{2+}, \mathrm{Si}(\mathrm{OH})_{4}$ and $\mathrm{Li}^{+}$, the incorporation of Laponite into bioinert materials led to enhanced cell adhesion by triggering interactions between the cell surface and Laponite [25,26]. Likewise, Gaharwar et al. reported that Laponite-enriched polycaprolactone electrospun webs induce osteogenic differentiation of human mesenchymal stem cells (hMSC) [27]. Schmidt and colleagues produced highly extensible nanocomposite nanofibers of poly(ethylene oxide) and Laponite for cell alignment [28]. As seen in the above examples, the incorporation of low Laponite content into bioinert polymeric networks drastically improves the bioactivity of the resultant materials. Thus, a similar improvement on the bioactivity of $\mathrm{CD}$ nanofibers can be expected with the incorporation of Laponite for their possible use in bio-related applications.

This paper reports the influence of Laponite clay on the polymerfree electrospinning of $\mathrm{CD}$ molecules and investigates the effect of the incorporated Laponite on the morphology of the resultant nanofibers. The CD/Laponite nanocomposite nanofibers with various Laponite contents were prepared by electrospinning (Fig. S1). Rheology was used to elucidate the changes in the flow behavior of CD solutions with the incorporation of Laponite. Conductivity measurements were performed to correlate the electrospinnability of $\mathrm{CD}$ solutions with increased conductivity due to the embedded Laponite clays. The CD/Laponite nanocomposite nanofibers were characterized by means of fiber morphology by SEM, chemical composition by EDX and XPS, Laponite distribution by TEM, mechanical and thermal properties by DMA and TGA, and structural analysis by WAXS.

\section{Results and discussion}

The aqueous mixtures of HP- $\beta$-CD and Laponite formed homogeneous dispersions. Below the concentration of $3.12 \mathrm{wt} \%$ Laponite, the solutions were clear and transparent, however, above the concentration of $3.12 \mathrm{wt} \%$ Laponite, turbid solutions were formed because of the formation of large aggregates in the solution (Fig. 1a). The solutions containing high Laponite contents formed highly viscous solutions, which did not flow like the solutions having lower Laponite contents once the vials were inverted (Fig. 1b). Due to the strong electrostatic interaction between CD molecules and Laponite, their solutions formed very viscous solutions. In electrospinning, the viscosity and conductivity of solutions are two critical factors that directly affect the formation, size and morphology of the resultant nanofibers. In this context, increasing solution conductivity generally produces thinner nanofibers. Because of the charged surface of Laponite particles (Fig. 1c, inset), the incorporation of Laponite increased conductivity with respect to Laponite content in the solution: the conductivity of a Laponite-free HP- $\beta$-CD solution was measured as $8.88 \mu \mathrm{S} / \mathrm{cm}$, while the HP- $\beta$-CD solution containing $0.62 \mathrm{wt} \%$ Laponite has conductivity of $16.43 \mu \mathrm{S} / \mathrm{cm}$. The presence of $3.12 \mathrm{wt} \%$ Laponite significantly increased conductivity almost three-fold to $46.26 \mu \mathrm{S} / \mathrm{cm}$ due to their aggregation caused by electrostatic interactions (Fig. 1c). A further rise in the Laponite content did not induce a marked increase on the solution conductivity $(48.29 \mu \mathrm{S} / \mathrm{cm}$ for $6.25 \mathrm{wt} \%$ Laponite solution). A similar increasing trend was also reported on the conductivity of Laponite particles in water by Jabbari-Farouji et al. [29]. The flow behavior of the HP- $\beta$-CD solutions containing various Laponite contents was explored by rheology, and the respective viscosity profiles of the solutions at an increasing shear rate were shown in Fig. 1d. The Laponite addition significantly increased viscosity at low shear rates $(\gamma)$, while the mixtures exhibited an unequalled degree of shear thinning at high shear rates (Fig. 1d). The zero-shear viscosity of the solution containing
$3.12 \mathrm{wt} \%$ Laponite was measured as $413 \mathrm{~Pa} \cdot \mathrm{s}$, and it drastically decreased to $0.6 \mathrm{Pas}$ with increasing $\gamma$ from 0.001 to $1000 \mathrm{~s}^{-1}$. This is consistent with the previous reports, which showed strong shear-thinning behavior of Laponite-containing solutions [30,31]. Even though the solutions show significant differences in zero-shear viscosity values $\left(\eta_{0}\right)$, electrostatic interactions between $\mathrm{CD}$ and Laponite become negligible at high shear rates (Fig. $1 \mathrm{~d}$, inset). The infinite viscosity $\left(\eta_{\infty}\right)$ of the HP- $\beta$-CD solutions increased with the incorporation of higher Laponite content (Fig. S2, see Supporting Information). However, this increase was insignificant with respect to the changes observed in the $\eta_{0}$. Further, a decreasing trend on $\dot{\gamma}_{\text {crit }}$ was observed with increasing Laponite content because of their shear-thinning behavior (Fig. 1d). The stress profiles of the respective solutions with increasing $\dot{\gamma}$ were also plotted (see Fig. S3, Supporting Information), where the solutions displayed unusual stress profiles at high Laponite loadings. In a normal case, the shear stress increases with the applied shear rate. But, as observed in Fig. S3, the stress first increases and passes through a maximum and drops before starting to rise again, suggesting that the solution at high shear rates exhibits a completely different profile than at low shear rates; Laponite clays align with the flow at high shear rates while they show so-called "house of cards" structure at low shear rates. Similar findings were also observed by McAlpine et al. in the mixture of organically-modified montmorillonite in methyl methacrylate/poly (methyl methacrylate) [32]. The authors attributed this behavior to the orientation of clays from the "house of cards" structure with increasing $\gamma$.

The morphology of the electrospun nanofibers and electrosprayed beads was explored through SEM analysis. Fig. 2 shows the SEM photos of the HP- $\beta$-CD nanofibers electrospun in the presence of various Laponite contents, together with Laponite-free nanofibers. The electrospinnability of $\mathrm{CD}$ molecules did not show any significant change with the incorporation of low laponite content. In the absence of Laponite, bead-free, smooth HP- $\beta$-CD nanofibers were formed with a mean diameter of $830 \pm 460 \mathrm{~nm}$ (Fig. 2a). The addition of $0.62 \mathrm{wt} \%$ Laponite decreased the diameter to $610 \pm 240 \mathrm{~nm}$ (Fig. 2b). Further increasing Laponite content to 1.56 and $3.12 \mathrm{wt} \%$ led to non-smooth nanofibers sizing $950 \pm 520$ and $690 \pm 410 \mathrm{~nm}$ in diameter, respectively (Fig. 2c, d), demonstrating the importance of electrostatic interactions between the charged surfaces of Laponite and the hydroxyl groups of HP- $\beta$-CD molecules on the fiber morphology. Increasing Laponite content to $6.25 \mathrm{wt} \%$ resulted in thinner fiber (mean diameter = $460 \pm 350 \mathrm{~nm}$ ) (Fig. 2e). One should note that the nanofibers produced in the presence of $0.62 \mathrm{wt} \%$ Laponite have round-shaped morphology while the nanofibers produced at 1.56 and $3.12 \mathrm{wt} \%$ have partial flattened domains so that an initial increase in the fiber diameter can be attributed to their morphology. Yet, in general, increasing Laponite content decreased the fiber diameter. Like the morphological deterioration of the nanofibers with increasing Laponite content, the flow behavior of the respective solutions shows significant differences on the profiles of shear-stress versus shear rate. The solutions with $3.12 \mathrm{wt} \%$ Laponite revealed a completely different profile than the solution containing lower Laponite contents (Fig. S3). Further, as revealed by the optical photos of the respective solutions (Fig. 1a, b), HP- $\beta$-CD solutions with 3.12 and $6.25 \mathrm{wt} \%$ Laponite shows no flow in an inverted position because of the presence of strong electrostatic interactions between the charged surface of Laponite and the backbone of $\mathrm{CD}$ molecules, which dominates the solution behavior.

The morphology of the nanofibers worsened with increasing Laponite content. The electrospinnability of the HP- $\beta$-CD significantly deteriorated for the solution containing $6.25 \mathrm{wt} \%$ Laponite (Fig. 2e). Increasing Laponite content led to non-smooth nanofibers because of their negative effect on the electrospinnability. Similar effect was also observed for nanocomposite nanofibers of a hydroxyapatite/chitosan system, in which the addition of the hydroxyapatite worsened the electrospinnability of chitosan [33]. Possible interactions between two precursors became very dominant and significantly increased the 
a
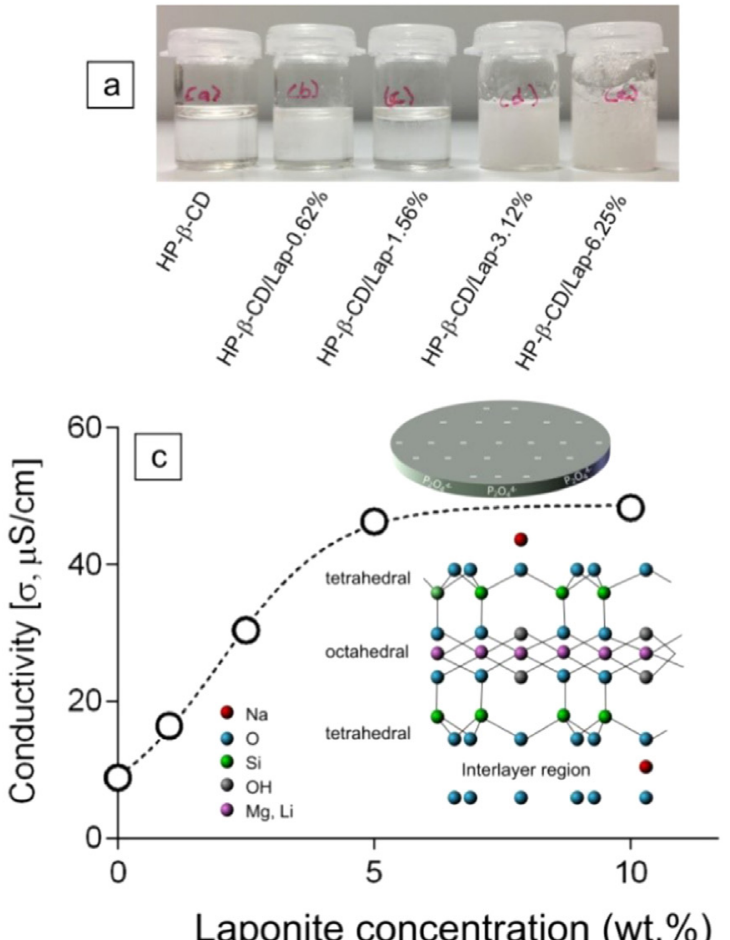
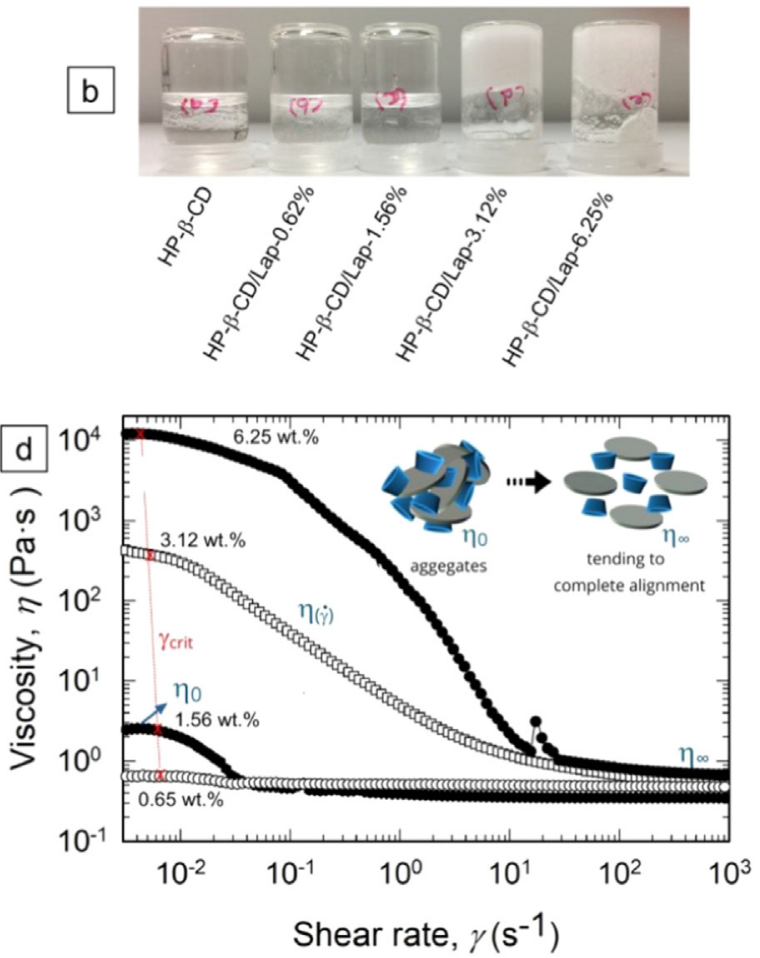

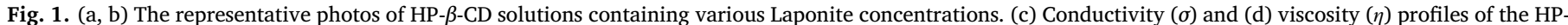

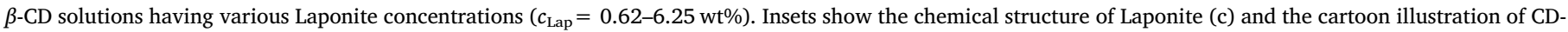
Laponite aggregates before and during flow (d).
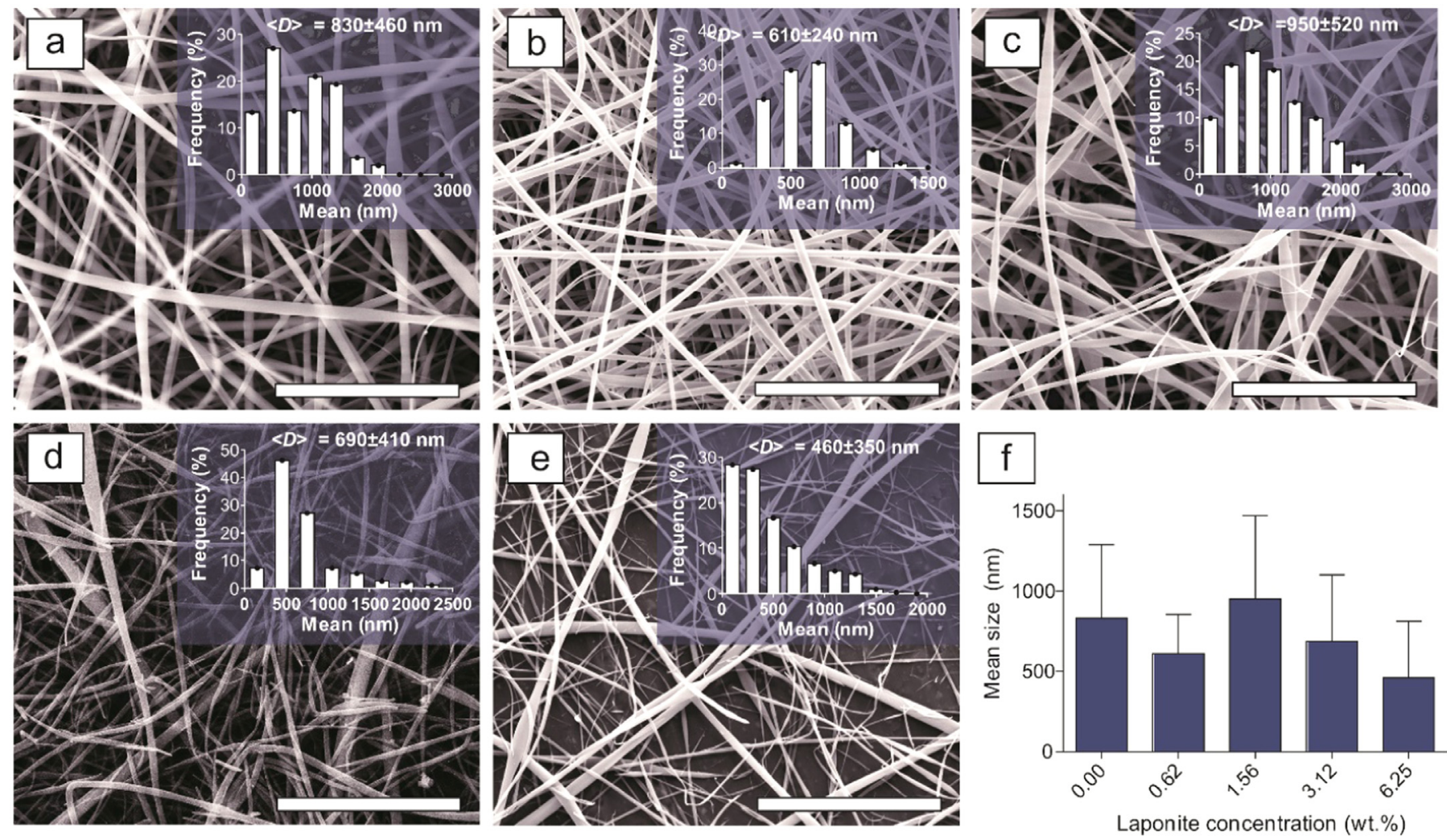

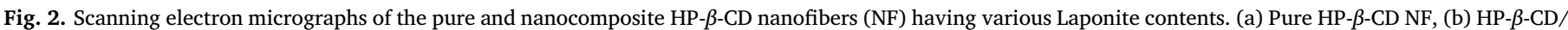

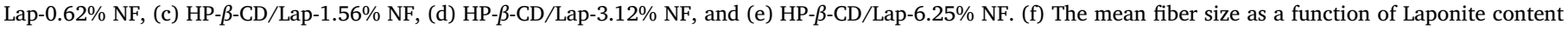
used. Insets (a-e) show the size distributions of the respective nanofibers. The scale bar denotes $20 \mu \mathrm{m}$. 

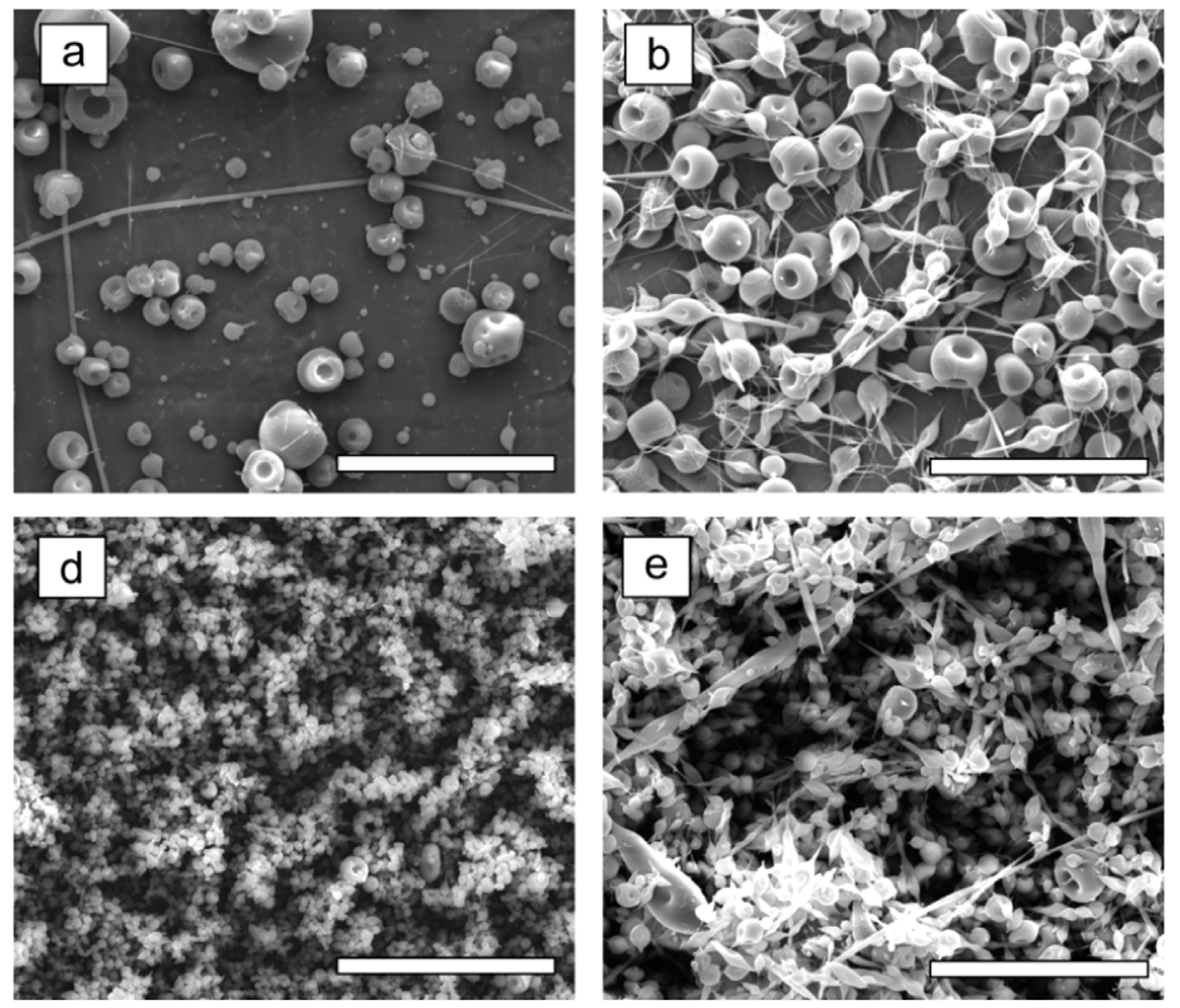

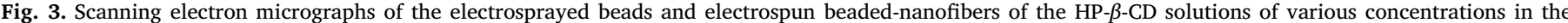

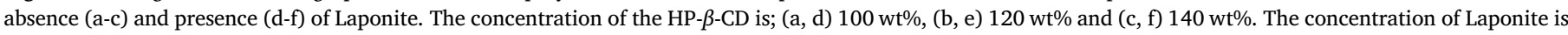
as follows; (d) $2.5 \mathrm{wt} \%$, (e) $2.1 \mathrm{wt} \%$ and (f) $1.8 \mathrm{wt} \%$. The scale bar denotes $20 \mu \mathrm{m}$.

solution viscosity. As shown in the Fig. $1 \mathrm{~d}$, the viscosity of the HP- $\beta$-CD drastically increased with higher Laponite content. Further boosting the Laponite concentration to $12.5 \mathrm{wt} \%$ resulted in beads due to the electrospraying of the composite solution (Fig. S4). The electrospraying can be attributed to the high viscosity of the respective solution (Fig. S5).

The electrospinning was also performed at the constant Laponite content with respect to water and various HP- $\beta$-CD concentrations (100-140 wt\%) (Fig. 3). Even though the incorporated Laponite significantly increased the solution viscosity, the mixed solution of $100 \mathrm{wt}$ $\%$ HP- $\beta$-CD and $1.8 \mathrm{wt} \%$ Laponite could not lead to the formation of nanofibers (Fig. 3d), as observed for the Laponite-free sample (Fig. 3a). Indeed, electrospraying has occurred because of the appearance of nanocomposite beads of Laponite and HP- $\beta$-CD. Further increasing HP$\beta$-CD concentration to $120 \mathrm{wt} \%$ could form extended beads for both Laponite-free HP- $\beta$-CD (Fig. 3b) and HP- $\beta$-CD solution containing $2.1 \mathrm{wt}$ $\%$ Laponite (Fig. 3e). The electrospinning of the Lap-free HP- $\beta$-CD solution containing $140 \mathrm{wt} \%$ HP- $\beta$-CD (Fig. $3 c$ ) and $140 \mathrm{wt} \%$ HP- $\beta$-CD solution having $1.56 \mathrm{wt} \%$ Laponite (Fig. $3 \mathrm{f}$ ) led to the formation of beaded fibers. The electrospinning of aqueous HP- $\beta$-CD solutions in the concentration range of $100-140 \mathrm{wt} \%$ could not form bead-free nanofibers regardless of Laponite presence. In brief, there was no morphological difference between Laponite-free HP- $\beta$-CD and HP- $\beta$-CD/Laponite systems. Even though the incorporation of Laponite in HP- $\beta$-CD has increased the solution viscosity significantly, our results suggested that the viscosity is not the only parameter for the formation of bead-free nanofibers from HP- $\beta$-CD/Laponite at lower HP- $\beta$-CD concentrations (i.e., 100-140 wt\%). Being a non-polymeric system, the electrospinnability of $\mathrm{CD}$ molecules is normally highly dependent on the intermolecular associations, i.e., viscosity. The conductivity of the respective solution plays a lesser important role on the electrospinnability but decreases fiber diameter with increasing conductivity. In the case of Laponite used as an additive, it drastically increases the solution viscosity due to electrostatic interactions between the charged surface of
Laponite and hydroxyl groups of CD molecules. This partially breaks hydrogen bonds among CDs when higher Laponite contents are used. But, for low Laponite contents, the electrospinnability of the composite mixtures of $\mathrm{CD}$ and Laponite does show any significant changes. We can clearly see this over the morphological change of the respective fibers with the incorporation of higher Laponite content. Whereas, no clear change on the fiber morphology could be detected for the electrospinning of CD solutions containing low Laponite contents (Fig. 3d,f).

The electrospinning of the HP- $\beta$-CD and HP- $\beta$-CD/Laponite solutions led to self-standing nanofibrous mats (Fig. 4a). Both mats (HP- $\beta$-CD and HP- $\beta$-CD/Laponite) could preserve their structure without any visible damage during folding, suggesting the structural integrity of $\mathrm{CD}$ mats despite their polymer-free nature (Fig. 4b, c). Laponite RDS has a chemical formula of $\mathrm{Na}_{0.7}^{+}\left[\left(\mathrm{Mg}_{5.5} \mathrm{Li}_{0.3}\right) \mathrm{Si}_{8} \mathrm{O}_{20}(\mathrm{OH})_{4}\right]^{-0.7}+\mathrm{Na}_{4} \mathrm{P}_{2} \mathrm{O}_{7}$ and comprises a significant amount of $\mathrm{Si}$ atoms that can be detected via EDX [34]. In this regard, the EDX spectra of the nanofibers produced at the constant HP- $\beta$-CD content and various Laponite contents (between 0.62 and $3.12 \mathrm{wt} \%$ ) revealed higher peak intensities for $\mathrm{Si}$ and $\mathrm{Mg}$ atoms because of the presence of higher embedded Laponite contents (Fig. 4d). Further, the EDX chemical mappings of the respective nanofibers confirmed the presence of Laponite by the appearance of Si atom in the nanofibers (Fig. 4d, insets).

The presence of Laponite in the nanofibers was also confirmed by XPS analysis, which allows the chemical composition of the material surface with a depth profile of ca. $10 \mathrm{~nm}$. We expect some Laponite on the fiber surface or fiber matrix near to the surface. XPS analysis was carried out on the samples having various Laponite contents (between 0.62 and $6.25 \mathrm{wt} \%)$. As Laponite is an oxygen-rich nanoparticle, the presence of Laponite should increase oxygen (O) content while decreasing carbon (C) ratio in the overall composition. The deconvoluted Si 2 X XPS spectra of the nanocomposite nanofibers showed a clear peak at $\sim 100 \mathrm{eV}$ due to silicon (Si), while the Laponite-free HP- $\beta$-CD nanofibers did not show up any peak in the respective region (Fig. S6). The 


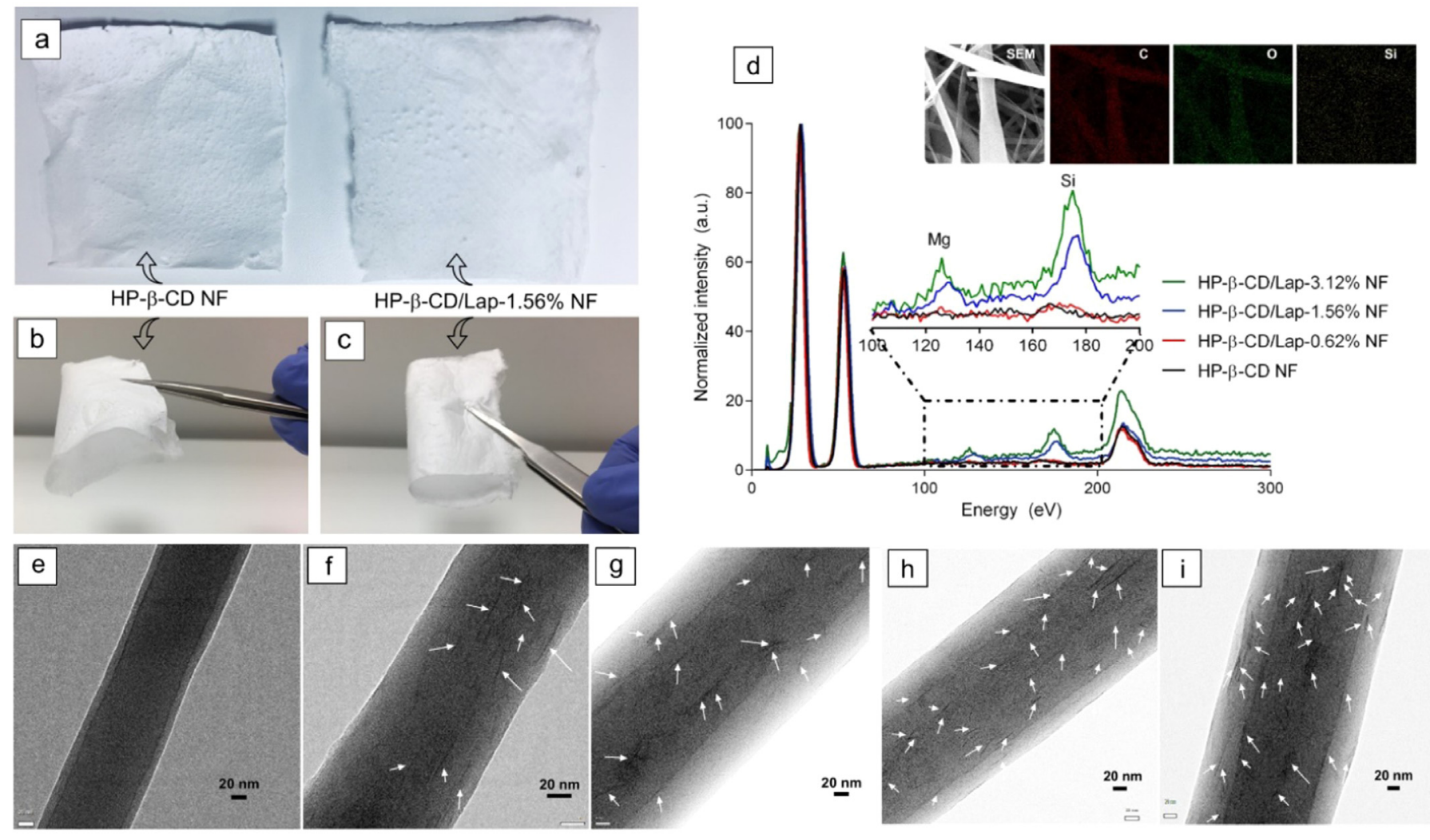

Fig. 4. (a) The representative photos of the pure and nanocomposite HP- $\beta$-CD mats ( $\left.c_{\text {Lap }}=1.56 \mathrm{wt} \%\right)$ collected on an aluminum foil $(8 \times 8 \mathrm{~cm})$. (b, c) The folding of the respective electrospun mats. (d) EDX spectra of the nanofibers of the HP- $\beta$-CD solutions containing various Laponite concentrations. Inset photos show the EDX chemical mappings of the nanocomposite nanofibers having $3.12 \mathrm{wt} \%$ Laponite for $\mathrm{C}, \mathrm{O}$ and $\mathrm{Si}$ atoms. Transmission electron micrographs (TEM) of the electrospun nanofibers (NF) of the HP- $\beta$-CD containing various Laponite contents. (e) HP- $\beta$-CD NF, (f) HP- $\beta$-CD/Lap $0.62 \%$ NF, (g) HP- $\beta$-CD/Lap $1.56 \%$ NF and (h) HP- $\beta$-CD/Lap $3.12 \% \mathrm{NF}$ and (i) HP- $\beta$-CD/Lap $6.25 \% \mathrm{NF}$. The arrows show Laponite clays in the fiber matrix.

atomic composition of the nanofibers was given in Table S1, where the pure HP- $\beta$-CD nanofiber sample is composed of carbon (C, $64.5 \%)$ and oxygen $(\mathrm{O}, 35.5 \%)$. On the other hand, the incorporation of $0.62 \mathrm{wt} \%$ Laponite decreased C content to $60.66 \%$ while increased O content to $39.34 \%$. Further increasing Laponite content to $6.25 \mathrm{wt} \%$, the respective values were found as 59.75 and $40.25 \%$, suggesting more Laponite incorporated into the fiber matrix.

The presence and distribution of the embedded Laponite in the HP$\beta$-CD nanofiber matrix were explored by TEM analysis, where the form of stacking/exfoliation of Laponite could be detected. Fig. 4(e-i) shows the TEM images of the HP- $\beta$-CD nanofibers with different Laponite loadings, in which they were homogeneously dispersed in the fiber matrix. Further, a high degree of orientation was observed along with the longitudinal axis due to shearing force during electrospinning. The respective thickness values for Laponite suggest their exfoliation within the fiber matrix because of the formation of homogeneous mixtures between HP- $\beta$-CD and Laponite. High shearing force disturbed their "house of cards" structure, as revealed by flow tests at high shear rates. The presence of higher Laponite content could be detected over TEM images, where more clay nanoparticles were observed with increasing Laponite content. At higher content of Laponite (i.e., 3.12 and $6.25 \mathrm{wt}$ $\%$ ), the presence of some non-exfoliated clay nanoparticles (i.e., layered Laponite) was also revealed (Fig. 4h, i).

Dynamic mechanical analyses of the fibers over tensile tests revealed a low degree of stretchability up to $4 \%$ due to their non-polymeric nature (Fig. S7). Furthermore, the fiber stretchability decreased with the incorporation of Laponite: the nanocomposite mats could be stretched up to $2 \%$. The young modulus of Laponite-free fibers was found as $1.63 \mathrm{MPa}$, while the addition of $0.62 \mathrm{wt} \%$ Laponite increased it to $2.60 \mathrm{MPa}$ (Fig. S7). Further increasing Laponite content to $1.56 \mathrm{wt} \%$, young modulus slightly decreased to 2.19 MPa. On the other hand, the addition of Laponite decreased tensile strength of the fibers; Laponitefree mat has a tensile strength of $42 \mathrm{kPa}$, while the nanocomposite mats have $23 \mathrm{kPa}$ and $31 \mathrm{kPa}$ for the fibers containing 0.62 and $1.56 \mathrm{wt} \%$ Laponite, respectively. Although the young moduli of the fibers increased with the Laponite incorporation, the stretchability and tensile strength of the fibers decreased with the addition of Laponite. This suggests that the incorporation of Laponite disturbed the hydrogen bonds between $\mathrm{CD}$ molecules and weakened intermolecular interactions, and therefore, the mechanical properties of the fibers reduced to some extent. Similar finding was observed over the formation of nonsmooth nanofibers with the incorporation of a higher Laponite content (Fig. 2). We also performed WAXS experiments for the structural analysis of pure Laponite, HP- $\beta$-CD nanofibers and HP- $\beta$-CD/Laponite nanocomposite nanofibers (Fig. S8). Due to the layered structures of Laponite, major diffraction peaks were appeared at $2 \theta$ of $6.9^{\circ}(d=$ $1.27 \mathrm{~nm}), 19.9^{\circ}(d=0.43 \mathrm{~nm}), 34.9^{\circ}(d=0.25 \mathrm{~nm})$ and $68.3^{\circ}(d=$ $0.15 \mathrm{~nm}$ ), while the pure HP- $\beta$-CD nanofibers, and HP- $\beta$-CD/Laponite nanocomposite nanofibers regardless of Laponite content, did not show any sharp peaks related to the Laponite clays. HP- $\beta$ - $C D$, unlike native $\mathrm{CD}$ molecules, is an amorphous molecule and does not show any sharp peak because of its amorphous structure. Hence, any peaks appear on the corresponding WAXS pattern for nanocomposite nanofibers can be attributed to the Laponite nanoparticles. In this regard, a small broad peak at $\sim 10^{\circ}$ was detected, and the peak intensity showed an increasing trend with an increasing Laponite content. This peak may be attributed to the shifting of the peak at $6.9^{\circ}$, which corresponds to a basal spacing of $1.27 \mathrm{~nm}$, suggests that the Laponite was largely exfoliated in the fiber matrix. After the electrospinning, Laponite was homogeneously distributed in the nanofibers as observed in the TEM analysis of the 
nanofibers, where only few layered Laponite could be detected once high Laponite content was used (Fig. 4h, i). Thermal analysis of the nanocomposite fibers was performed in the temperature range of $25-700{ }^{\circ} \mathrm{C}$ (Fig. S9). To minimize the influence of water content, TGA curves were normalized at $100^{\circ} \mathrm{C}$. The Laponite-free sample showed a mass loss above $300{ }^{\circ} \mathrm{C}$, which could be attributed to the decomposition of HP- $\beta$-CD molecules [35]. The incorporation of Laponite did not show any significant influence on the thermal stability of the fibers, however, Laponite-containing sample showed an increased remaining mass at $700{ }^{\circ} \mathrm{C}$ because of the embedded Laponite. Laponite particles are thermostable and do not show a mass loss except the loss of the adsorbed water molecules in the respective temperature range.

\section{Conclusion}

Polymer-free HP- $\beta$-CD nanofibers with various Laponite contents were successfully produced through electrospinning. The incorporation of Laponite into the aqueous solutions of the HP- $\beta$-CDs significantly increased the zero-shear viscosity of the HP- $\beta$-CD solutions. The incorporation of a low Laponite content $(0.62 \mathrm{wt} \%)$ induced smooth nanofibers, while higher Laponite content ( $\geq 1.56 \mathrm{wt} \%$ ) led to nonsmooth nanofibers. On the other hand, the use of a very high Laponite content $(12.5 \mathrm{wt} \%)$ led to the electrosprayed beads. The presence of the embedded Laponite in the nanofibers was confirmed over chemical analysis by EDX and XPS. The TEM analysis of the nanofibers revealed homogeneous distribution of Laponite in the matrix due to their exfoliation caused by the shearing force of electrospinning. The exfoliation of Laponite particles was also confirmed over the WAXS patterns of the samples, which revealed the absence of diffraction patterns of Laponite. The nanocomposite nanofiber mats having low Laponite contents led to self-standing and flexible nanofibrous materials as revealed by DMA. The thermal analysis of the nanofibers revealed a slight increase in the thermal stability of the CD fibers with the incorporation of Laponite. Overall, this study, for the first time, report the electrospinning of polymer-free nanocomposite nanofibers from HP- $\beta$-CD and Laponite, and such fibers with low Laponite contents may find applications as sorbent materials due to affinity of $\mathrm{CD}$ molecules to organic micropollutants and Laponite towards heavy metals. Such materials can also be exploited for biological applications due to the intrinsic bioactivity of the embedded Laponite clays.

\section{Appendix A. Supporting information}

Supplementary data associated with this article can be found in the online version at doi:10.1016/j.coco.2018.12.002.

\section{References}

[1] J. Zeng, X. Xu, X. Chen, Q. Liang, X. Bian, L. Yang, X. Jing, Biodegradable electrospun fibers for drug delivery, J. Control. Release 92 (3) (2003) 227-231.

[2] B. Ding, M. Wang, X. Wang, J. Yu, G. Sun, Electrospun nanomaterials for ultrasensitive sensors, Mater. Today 13 (11) (2010) 16-27.

[3] M. Stasiak, A. Studer, A. Greiner, J.H. Wendorff, Polymer fibers as carriers for homogeneous catalysts, Chem. - Eur. J. 13 (21) (2007) 6150-6156.

[4] A. Celebioglu, F. Topuz, T. Uyar, Water-insoluble hydrophilic electrospun fibrous mat of cyclodextrin-epichlorohydrin polymer as highly effective sorbent, ACS Appl. Polym. Mater. (2018), https://doi.org/10.1021/acsapm.8b00034.

[5] T. Uyar, E. Kny, Electrospun Materials for Tissue Engineering and Biomedical Applications: Research, Design and Commercialization, Elsevier, Woodhead Publishing, 2017.

[6] D.I. Braghirolli, D. Steffens, P. Pranke, Electrospinning for regenerative medicine: a review of the main topics, Drug Discov. Today 19 (6) (2014) 743-753.

[7] T. Jiang, E.J. Carbone, K.W.H. Lo, C.T. Laurencin, Electrospinning of polymer nanofibers for tissue regeneration, Prog. Polym. Sci. 46 (2015) 1-24.

[8] S. Jiang, Y. Chen, G. Duan, C. Mei, A. Greiner, S. Agarwal, Electrospun nanofiber reinforced composites: a review, Polym. Chem. 9 (20) (2018) 2685-2720.
[9] M.G. McKee, J.M. Layman, M.P. Cashion, T.E. Long, Phospholipid nonwoven electrospun membranes, Science 311 (5759) (2006) 353-355.

[10] M.P. Cashion, X. Li, Y. Geng, M.T. Hunley, T.E. Long, Gemini surfactant electrospun membranes, Langmuir 26 (2) (2010) 678-683.

[11] A. Celebioglu, T. Uyar, Cyclodextrin nanofibers by electrospinning, Chem. Commun. 46 (37) (2010) 6903-6905.

[12] A. Celebioglu, T. Uyar, Electrospinning of nanofibers from non-polymeric systems: polymer-free nanofibers from cyclodextrin derivatives, Nanoscale 4 (2) (2012) 621-631.

[13] J.L. Manasco, C.D. Saquing, C. Tang, S.A. Khan, Cyclodextrin fibers via polymerfree electrospinning, RSC Adv. 2 (9) (2012) 3778-3784.

[14] W. Zhang, M. Chen, B. Zha, G. Diao, Correlation of polymer-like solution behaviors with electrospun fiber formation of hydroxypropyl- $\beta$-cyclodextrin and the adsorption study on the fiber, PCCP 14 (27) (2012) 9729-9737.

[15] M. Allais, D. Mailley, P. Hebraud, D. Ihiawakrim, V. Ball, F. Meyer, A. Hebraud, G. Schlatter, Polymer-free electrospinning of tannic acid and cross-linking in water for hybrid supramolecular nanofibres, Nanoscale 10 (2018) 9164-9173.

[16] A. Celebioglu, T. Uyar, Electrospinning of nanofibers from non-polymeric systems: electrospun nanofibers from native cyclodextrins, J. Colloid Interface Sci. 404 (2013) 1-7.

[17] A. Celebioglu, T. Uyar, Electrospun gamma-cyclodextrin ([gamma]-CD) nanofibers for the entrapment of volatile organic compounds, RSC Adv. 3 (45) (2013) $22891-22895$

[18] A. Celebioglu, T. Uyar, Electrospinning of polymer-free nanofibers from cyclodextrin inclusion complexes, Langmuir 27 (10) (2011) 6218-6226.

[19] F. Kayaci, H.S. Sen, E. Durgun, T. Uyar, Functional electrospun polymeric nanofibers incorporating geraniol-cyclodextrin inclusion complexes: high thermal stability and enhanced durability of geraniol, Food Res. Int. 62 (2014) 424-431.

[20] D. Su, L. Jiang, X. Chen, J. Dong, Z. Shao, Enhancing the gelation and bioactivity of injectable silk fibroin hydrogel with laponite nanoplatelets, ACS Appl. Mater. Interfaces 8 (15) (2016) 9619-9628.

[21] T. Ahlfeld, G. Cidonio, D. Kilian, S. Duin, A.R. Akkineni, J.I. Dawson, S. Yang, A. Lode, R.O.C. Oreffo, M. Gelinsky, Development of a clay based bioink for 3D cell printing for skeletal application, Biofabrication 9 (3) (2017) 16.

[22] D. Shi, T. Mei, Q. Chen, F. Duan, M. Chen, Preparation and adsorption properties of laponite-cyclodextrin complex, Mater. Res. Innov. 19 (suppl 9) (2015) S9-157-S9161.

[23] P.C. Thomas, B.H. Cipriano, S.R. Raghavan, Nanoparticle-crosslinked hydrogels as a class of efficient materials for separation and ion exchange, Soft Matter 7 (18) (2011) 8192-8197.

[24] S. Wang, R. Castro, X. An, C. Song, Y. Luo, M. Shen, H. Tomas, M. Zhu, X. Shi, Electrospun laponite-doped poly(lactic-co-glycolic acid) nanofibers for osteogenic differentiation of human mesenchymal stem cells, J. Mater. Chem. 22 (44) (2012) 23357-23367.

[25] A. Hoppe, N.S. Güldal, A.R. Boccaccini, A review of the biological response to ionic dissolution products from bioactive glasses and glass-ceramics, Biomaterials 32 (11) (2011) 2757-2774.

[26] F. Topuz, A. Nadernezhad, O.S. Caliskan, Y.Z. Menceloglu, B. Koc, Nanosilicate embedded agarose hydrogels with improved bioactivity, Carbohydr. Polym. 201 (2018) 105-112.

[27] A.K. Gaharwar, S. Mukundan, E. Karaca, A. Dolatshahi-Pirouz, A. Patel, K. Rangarajan, S.M. Mihaila, G. Iviglia, H. Zhang, A. Khademhosseini, Nanoclayenriched poly( $\varepsilon$-caprolactone) electrospun scaffolds for osteogenic differentiation of human mesenchymal stem cells, Tissue Eng. Part A 20 (15-16) (2014) 2088-2101.

[28] A.K. Gaharwar, P.J. Schexnailder, A. Dundigalla, J.D. White, C.R. Matos-Pérez, J.L. Cloud, S. Seifert, J.J. Wilker, G. Schmidt, Highly extensible bio-nanocomposite fibers, Macromol. Rapid Commun. 32 (1) (2010) 50-57.

[29] S. Jabbari-Farouji, H. Tanaka, G.H. Wegdam, D. Bonn, Multiple nonergodic disordered states in Laponite suspensions: a phase diagram, Phys. Rev. E 78 (6) (2008) 061405.

[30] A.K. Gaharwar, R.K. Avery, A. Assmann, A. Paul, G.H. McKinley, A. Khademhosseini, B.D. Olsen, Shear-thinning nanocomposite hydrogels for the treatment of hemorrhage, ACS Nano 8 (10) (2014) 9833-9842.

[31] S.A. Wilson, L.M. Cross, C.W. Peak, A.K. Gaharwar, Shear-thinning and thermoreversible nanoengineered inks for 3D bioprinting, ACS Appl. Mater. Interfaces 9 (50) (2017) 43449-43458.

[32] M. McAlpine, E. Hudson Nicholas, J. Liggat John, A. Pethrick Richard, D. Pugh, I. Rhoney, Study of the factors influencing the exfoliation of an organically modified montmorillonite in methyl methacrylate/poly(methyl methacrylate) mixtures, J. Appl. Polym. Sci. 99 (5) (2005) 2614-2626.

[33] Y. Zhang, J.R. Venugopal, A. El-Turki, S. Ramakrishna, B. Su, C.T. Lim, Electrospun biomimetic nanocomposite nanofibers of hydroxyapatite/chitosan for bone tissue engineering, Biomaterials 29 (32) (2008) 4314-4322.

[34] F. Topuz, M. Bartneck, Y. Pan, F. Tacke, One-step fabrication of biocompatible multifaceted nanocomposite gels and nanolayers, Biomacromolecules 18 (2) (2017) 386-397.

[35] S. Shang, K.-L. Chiu, S. Jiang, Synthesis of immobilized poly(vinyl alcohol)/cyclodextrin eco-adsorbent and its application for the removal of ibuprofen from pharmaceutical sewage, J. Appl. Polym. Sci. 134 (21) (2017). 